\title{
Free and serial recall of pictures, sounds, and words
}

\author{
ALLAN PAIVIO \\ University of Western Ontario, London, Ontario, Canada \\ RONALD PHILIPCHALK \\ St. Thomas University, Chatham, New Brunswick, Canada \\ and \\ EDWARD J. ROWE \\ Memorial University of Newfoundland, St. John's, Newfoundland, Canada

\begin{abstract}
Pictures and sounds of familiar objects and their visual and auditory names were compared in free and serial recall tasks. The results of two experiments showed, most notably, that type of task interacted significantly with symbolic (verbal-nonverbal) and sensory (visual-auditory) modalities. Nonverbal items were remembered relatively better in free recall, whereas verbal items were superior in serial recall. In the visual modality, pictures were superior to words in both recall tasks; in the auditory modality, conversely, nonverbal sounds were inferior to words in serial recall but sounds and words did not differ in free recall. The results indicate that a satisfactory general theory of memory must encompass distinctions in both symbolic and sensory modalities as well as differences in the organizational demands of the memory task.
\end{abstract}

Philipchalk and Rowe (1971) reported an experiment involving easily recognizable environmental sounds and their verbal labels as stimuli in serial and free recall learning. Correct verbal recall of the two types of items did not differ in free recall, but words were significantly better than sounds in serial recall. The results for serial recall are comparable in one respect to results obtained by Warren, Obusek, Farmer, and Warren (1969). They found that subjects were unable to report the order of four sounds (hiss, buzz. high tone. low tone) presented in rapid succession. although they had no difficulty recognizing the individual sounds.

The results of the different studies suggest that sequential memory is poorer for discrete nonverbal sounds than it is for speech sounds, but memory for the items themselves is not similarly affected. The results can be explained by assuming that rapid verbal coding during input is necessary for storing or retrieving order information, and that such coding is more difficult in the case of the sounds than auditory words. The items themselves, however, can be retained in, and the appropriate verbal label retrieved from, either code during the recall test. Such a dual coding approach to memory for verbal and nonverbal stimuli has been proposed by Paivio (1971). The dual coding theory has been further supported by selective interference effects (Rowe, Philipchalk, \& Cake, 1974: Rowe \& Rowe, 1975), recall organization

This research was supported by Grant $\mathbf{A 0 0 8 7}$ from the National Research Council of Canada. Requests for reprints should be sent to Allan Paivio, Department of Psychology. University of Western Ontario, London, Ontario, Canada N6A SC2.
(Philipchalk \& Cyr, Note 1), continuous recognition (Philipchalk, Note 2), and immediate sequential memory (Rowe, 1974) involving words and sounds as stimuli.

The sound-word data and their dual-coding interpretation are consistent also with results from comparisons involving pictures and words as memory stimuli. Numerous studies have demonstrated that free recall is higher for pictures than for their concrete noun labels (for a summary, see Paivio, 1971). Paivio and Csapo (1969) found, however, that pictures did not surpass words in serial recall or immediate memory span even at a presentation rate that presumably permitted implicit labeling of the stimuli. Moreover. pictures were inferior to words in both sequential tasks (but not in free recall) at a fast presentation rate, which presumably prevented implicit labeling during input (cf. Del Castillo \& Gumenik, 1972). Thus comparisons of words with both pictures and sounds are consistent with the hypothesis that the verbal memory system is specialized for storing sequential order as well as item information, whereas nonverbal memory (imagery) is effective for storage of items but not their order. Since this difference between verbal and nonverbal memory occurred for both verbal and auditory stimuli, it can be viewed as a functional distinction between symbolic memory systems, rather than sensory modalities of memory (cf. Paivio. 1972).

While the general conclusions apply to both sensory modalities, the relative levels of verbal and nonverbal recall appear to differ for visual and auditory stimuli. In the Paivio and Csapo (1969) experiment, pictures 
Table 1

Mean Total Serial and Free Recall Scores Over Six Trials for Four Types of Items: Experiment I

\begin{tabular}{lrrrr}
\hline & Pictures & Sounds & $\begin{array}{c}\text { Words } \\
\text { (V) }\end{array}$ & $\begin{array}{c}\text { Words } \\
\text { (A) }\end{array}$ \\
\hline Serial & & & & \\
$\quad$ Mean & 43.3 & 46.9 & 51.2 & 49.4 \\
SD & 11.2 & 12.8 & 9.9 & 8.3 \\
Free & & & & \\
Mean & 59.0 & 57.1 & 58.3 & 52.5 \\
SD & 5.1 & 5.6 & 6.0 & 6.2 \\
\hline
\end{tabular}

surpassed concrete words in free recall, but the two types of stimuli did not differ in serial recall at the slower of two presentation rates. On the other hand, Philipchalk and Rowe (1971) found that sounds and concrete words did not differ in free recall, but sounds were inferior to words in serial recall. Thus it appears that pictures and sounds interact similarly with their verbal counterparts in affecting free and serial recall, but the general level of recall (relative to words) is higher for pictures than for sounds. This observation, if reliable, is important because it implies that visual memory is superior to auditory memory in the case of concrete stimuli but not words. In fact, auditory presentation generally results in higher recall than visual presentation of verbal items in certain short-1erm memory tasks (see Murdock, 1967).

The apparent differences in recall for pictures and sounds, however, are observations from different experiments. Since those experiments differed on such variables as presentation rate. codability of items. length of list. etc., the inferences may not be justified. The primary purpose of the present experiments was to compare pictures and their visually presented labels with sounds and their spoken mames under more carefully controlled conditions.

\section{EXPERIMENT 1}

\section{Method}

Materials. The stimuli were line drawings, sounds, and verbal labels for 12 tamiliar objects. The 12 items were selected from a larger pool of approximately 120 items, for the representative sounds of which verbal labeling data had been gathered. The sounds chosen had been labeled consistently by at least 16 of a normative group of 20 subjects. For the present experiment, each sound was rerecorded at a duration approximating its mean labeling latency (obtained from the same group of 20 subjects) plus one standard deviation. The sounds ranged in duration from 2.0 to $4.5 \mathrm{sec}$, with a mean of 3.5 . The interval between sounds was $1.0 \mathrm{sec}$. The words used in the verbal conditions were the labels of the sounds-telephone, train. clock, horse, drum, saw, dog, hammer, car, bird, baby, and rooster. The words were recorded by a male speaker at a $4.5-\mathrm{sec}$ rate, which was the average rate of presentation of the sounds. In the visual presentation condition, the words were printed in block capitals and presented as negative slides. The pictures were simple line drawings of the objects represented by the sounds and words. These were also presented as negative slides. The presentation rate for the visual material was $4.5 \mathrm{sec}$, consisting of a 3.5 -sec presentation and a 1 -sec blank interval.
Procedure. Each subject learned only one kind of material under either a free recall or a serial recall condition. They were tested individually or in pairs in a small room. Each subject was seated at a table; if two subjects were tested simultaneously, a screen was placed between them. The auditory material was presented through headphones, using a Sony tape recorder. The subjects first heard the appropriate instructions, which were also recorded on the tape, regarding the type of material that would be presented (words or sounds) and the way in which the items were to be recalled (free or serial). For the sounds, the instructions requested that the subject try to use a single word to label each sound in recall. Six trials were given. The items were presented in a different random order on each trial for the tree recall conditions; a constant order was used for each trial of serial recall. After each presentation of the list, $30 \mathrm{sec}$ were allowed for written recall. The subjects wrote their answers on prepared answer sheets, which were collected at the end of each trial. The visual items were projected by a Carousel slide projector on a small screen at the front of the room. The instructions and recall procedure paralleled that for the auditory material.

Subjects. The subjects were 96 volunteers from undergraduate psychology courses. They were assigned in rotation to the cells of the 2 by 2 by 2 independent-measures design, with the restriction that the number of males in each cell be approximately equal.

\section{Results and Discussion}

The mean total number of items recalled correctly (in their correct serial positions in the case of serial recall) over the six trials are presented in Table 1 . The data were analyzed by a 2 by 2 by 2 by 6 analysis of variance, with task (free, serial), symbolic modality (verbal. nonverbal), sensory modality (visual, auditory), and trials as factors. Trials was the only repeated measure.

A significant main effect showed that free recall scores were generally higher than serial recall scores $[F(1,88)=24.46, \mathrm{p}<.001]$. This was qualified by a Task by Trials interaction $[F(5,44)=10.80$. $p<.001]$, which indicated that the inferiority of serial recall relative to free recall scores decreased over trials. Trials also had a significant main effect and entered into an uninterpretable triple interaction. These effects are of no theoretical interest here, so they will not be discussed.

The most important finding from the experiment was the interaction of Task by Symbolic Modality $[F(1.88)=4.56 . p<.05]$, shown in Figure 1. It can be seen than nonverbal stimuli were somewhat better than words in free recall. but inferior to words in

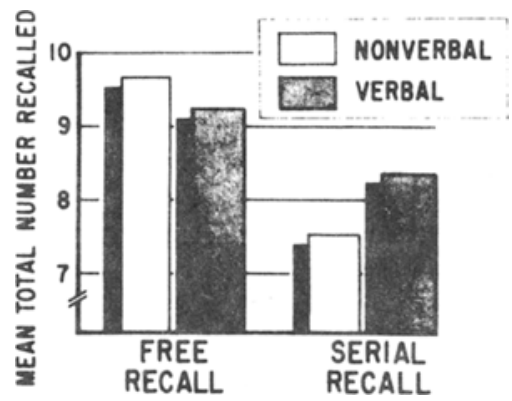

Figure 1. Recall as a function of type of task (free vs. serial recall) and material (verbal vs. nonverbal) in Experiment $I$. 
serial recall. In general. these results are consistent with previous experiments on the problem (Paivio \& Csapo, 1969; Philipchalk \& Rowe, 1971) and support the theory that the verbal memory system is specialized for sequential representation of items but nonverbal memory is not. The verbal items presumably had more direct access to the verbal memory system than did the nonverbal items, despite the fact that the latter were selected so as to be easily named implicitly during input at the slow rate of presentation used in the study. In the case of item recall, however, the evidence suggests that nonverbal memory is as good as or superior to verbal memory.

Despite the general agreement, some aspects of the results are somewhat inconsistent with previous ones in the area. Free recall has usually been found to be significantly better for pictures than for their verbal labels, but the difference was very slight in the present study (means over six trials $=9.83$ and 9.72 ). This might be due to the unusually slow rate of presentation and the relatively short list used here. The slow rate would facilitate verbal coding of pictures as well as image arousal to words, thereby tending to reduce any coding differences that otherwise might have occurred. The other suggestive discrepancy is that sounds tended to surpass auditory words in free recall $(\overline{\mathrm{x}}=9.51$ and 8.75$)$, whereas this did not occur in the Philipchalk and Rowe (1971) study. This difference is also small, however, and overshadowed by the general consistent pattern of results in the two experiments.

\section{EXPERIMENT II}

Experiment II was essentially a replication of Experiment I. with the major change being that the list length was increased from 12 to 20 items of each type. The presentation rate for the different stimuli was also changed slightly.

\section{Method}

The types of stimuli and the procedure were generally the same as in Experiment 1 . The 20 verbal items were dog, telephone, drum, saw, horse, hammer, car, train, baby, rooster, typewriter, tires, motorcycle, bagpipes. lion, rain, pigs, door, bell, and elephant. The characteristics of the corresponding pictures and environmental sounds were as in Experiment I. The presentation time for sounds ranged from 1.60 to $5.90 \mathrm{sec}$, and the interval between sounds was $1.0 \mathrm{sec}$, for a mean of $4.01 \mathrm{sec}$. The rate was $4.2 \mathrm{sec}$ for auditory words and $4 \mathrm{sec}$ for pictures and visual words. Because of the increased number of items, the recall time was increased to $1 \mathrm{~min}$ for all conditions. After the final recall trial. subjects in the picture and sound conditions were presented the stimulus items again and were asked to write the word they had used to label each item during the learning task. These subject-provided labels were used in scoring both free and serial recall.

The subjects were 160 undergraduate students who were paid $\$ 2$ each for their participation in the experiment. They were assigned randomly to one of the eight experimental conditions in groups ranging from 1 to 10 , with a total of 20 subjects in each condition.
Table 2

Mean Total Serial and Free Recall Scores Over Six Trials for Four Types of Items: Experiment II

\begin{tabular}{lcccc}
\hline & Pictures & Sounds & $\begin{array}{c}\text { Words } \\
\text { (V) }\end{array}$ & $\begin{array}{c}\text { Words } \\
\text { (A) }\end{array}$ \\
\cline { 1 - 1 } Serial & & & & \\
$\quad$ Mean & 79.1 & 63.4 & 73.3 & 77.5 \\
$\quad$ SD & 15.8 & 10.7 & 14.4 & 17.0 \\
Free & & & & \\
$\quad$ Mean & 103.7 & 95.4 & 92.2 & 94.0 \\
$\quad$ SD & 9.8 & 11.0 & 12.2 & 9.3 \\
\hline
\end{tabular}

\section{Results and Discussion}

Serial recall was again scored strictly, so that an item was correct only if it appeared in its appropriate serial position on the recall sheet. These data were analyzed by a 2 (free vs. serial) by 2 (verbal vs. nonverbal) by 2 (auditory vs. visual) by 6 (trials) analysis of variance. Trials again yielded a significant main effect and an interaction with task, as in Experiment 1. These effects are of no theoretical interest here. The data were also analyzed using a lenient scoring system for serial recall in which a correct sequence of two or more items was scored as correct although their absolute ordinal positions were incorrect. The major effect of the lenient scoring was to raise serial recall scores for early trials, particularly in the case of the nonverbal sounds. This resulted in the following departures from those reported below for the strict analysis: the superiority of visual over auditory presentation fell short of significance $(F=$ 3.54), and the significant double interaction of sensory modality by symbolic mode was slightly qualified by trials $(p<.05)$. Since these modifications did not qualify the major conclusions reported here, only the results of the strict analysis are reported in detail.

The results of interest involve mean recall scores collapsed over trials. These are presented in Table 2 for the four types of stimuli and the two tasks. The analysis of variance revealed the following significant main effects: free recall was superior to serial recall $[F(1,152)=134.4, p<.01]$, and visual presentation was superior to auditory $[F(1,152)=4.15, p<.05]$. Several interactions were also significant. As in the previous experiments. task interacted with symbolic modality $[F(1,152)=5.31, p<.05]$, so that nonverbal items were better in free recall, while verbal items were better in serial recall (Figure 2). Sensory modality interacted with symbolic mode $[F(1,152)=$ 15.02. $\mathrm{p}<.01]$, so that nonverbal items were recalled better than verbal items under visual presentation, whereas the reverse was true for auditory presentation (Figure 3).

The results were analyzed further by separate analyses of variance for auditory and visual presentation. The analysis of auditory presentation 


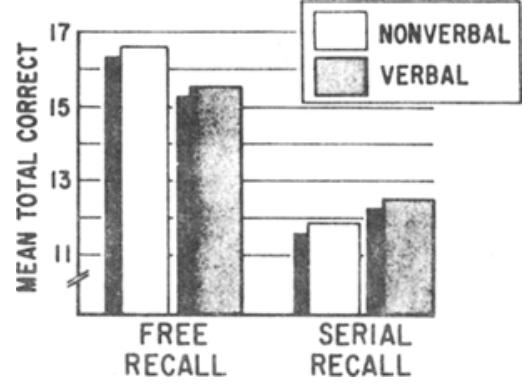

Figure 2. Recall as a function of type of task (free vs. serial) and material (verbal vs. nonverbal) in Experiment II.

showed that free recall was superior to serial recall $[F(1,76)=80.88, p<.01]$ and verbal items were superior to nonverbal $[\mathrm{F}(1,76)=6.92, \mathrm{p}<.02]$. Task and modality also interacted $[\mathrm{F}(1,76)=5.84$, $p<.02$ ], so that serial recall of words was superior to serial recall of sounds, but the two did not differ in free recall. These results confirm Experiment I and Philipchalk and Rowe (1971).

The analysis of visually presented materials showed once again that free recall was superior to serial recall $[F(1,76)=55.76, p<.01]$. In addition, pictures were recalled better than words $[F(1,76)=8.10, \mathrm{p}<.01]$. The predicted interaction of Task by Symbolic Modality did not approach significance under visual presentation conditions $(\mathrm{F}<.81)$.

\section{DISCUSSION}

The results of both experiments showed generally that nonverbal items were remembered better in free recall whereas verbal items were better in serial recall. The important point to note is that this interaction effect involves a distinction in symbolic modality (i.e., verbal vs. nonverbal) rather than sensory modality. Whether visual or auditory, nonverbal stimuli show the greater decrement (relative to words) when sequential order must be stored along with item information. These findings cannot be handled by memory theories that emphasize verbal processes alone. Nor is it sufficient to supplement such verbal

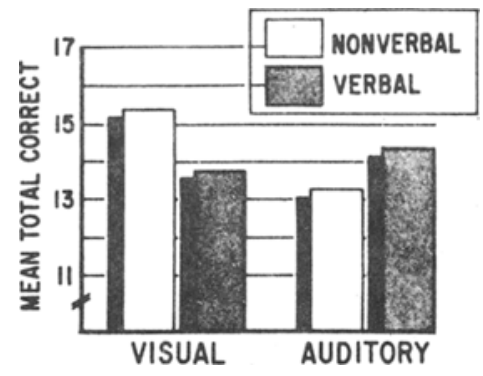

Figure 3. Recall as a function of visual vs. auditory presentation and verbal vs. nonverbal material in Experiment $\mathbf{I}$. theories by referring to distinctions in the sensory modality of verbal input, although such distinctions are obviously relevant to memory performance. Instead, a satisfactory general theory of memory must encompass distinctions in both symbolic and sensory modalities, and qualify any effects attributed to either in terms of the type of item organization required in the memory task. The present experiment and previous related ones support the hypothesis that nonverbal items are stored at least as efficiently as verbal items, but sequential information is more effectively retained in the verbal mode.

Experiment II suggested further that the symbolic modality effects are qualified by sensory modality. Thus, pictures were superior to visual words in both free and serial recall. Conversely, nonverbal sounds were clearly inferior to auditory words in serial recall but sounds and words did not differ in free recall. These results tend to confirm the observations in different experiments in the literature, namely, that superiority of memory for nonverbal as compared to verbal stimuli is found only in the visual modality. In the case of auditory stimuli, nonverbal sounds either do not surpass auditory words or are inferior to them, depending on the task. These differences could be due either to the spatially integrated properties of - pictures, which makes them more memorable than sounds or words, or to a greater ease of dual coding in the case of pictures than sounds or words. Mnemonic superiority of nonverbal images and dual coding are both implicated in memory for pictures (e.g., Paivio \& Csapo, 1973), but the relative contributions of nonverbal memory per se and of dual coding is less clear in the case of memory for sounds.

Comparisons of the different kinds of stimuli is complicated by the fact that environmental sounds vary greatly in the presentation time necessary for their identification whereas pictures and words do not. The problem was handled in the present experiments by varying the duration of sounds so that each was presented long enough to be identified and implicitly labeled. The pictures and words were, however, presented at a consistent rate approximately equal to the mean rate for the sounds. This introduced two problems. First, there would be less time for elaborative coding and rehearsal of sounds that were presented at rates less than the average than for the corresponding pictures or words. This would not account for the important finding that sounds were inferior to words only in serial recall, but it could explain part of the superiority of pictures over sounds in Experiment II. Such an explanation implies that recall scores for sounds should correlate positively with their duration. This possibility was checked by computing average recall scores for each of the 20 sounds used in Experiment II and correlating these with the exposure durations of the sounds. This was done for each trial for both recall tasks. The resulting 
correlations were all nonsignificant, ranging from .31 to -.21 in the case of free recall and from -.02 to -.19 in the case of serial recall. Thus, variation in sound duration did not appear to be an important factor in the recall of individual sounds. The second problem is that the irregular rate of presentation might have had a different effect on free recall and serial recall of sounds, e.g., serial recall of the list as a whole might suffer relatively more from irregular temporal pacing. This possibility remains to. be investigated.

\section{REFERENCE NOTES}

1. Philipchalk. R. P.. \& Cyr, J. Organization and free recall of meaningful sounds and words. Presented at the Annual Meeting of the Canadian Psychology Association, 1974.

2. Philipchalk, R. P. Memory for familiar sounds and their labels. Presented at the Annual Meetings of the Psychonomic Society. 1972.

\section{REFERENCES}

Del Castillo, D. M., \& Gumenik, W. E. Sequential memory for familiar and unfamiliar forms. Journal of Experimental Psychology, 1972, 95, 90-96.

Murdock, B. B., JR. Recent developments in short-term memory. British Journal of Psychology, 1967, 58, 421-433.
Paivio, A. Imary and verbal processes. New York: Holt, Rinehart, and Winston, 1971.

Paivio, A. Symbolic and sensory modalities of memory. In M. E. Myer (Ed.), The third Western symposium on learning: Cognitive leaming. Bellingham: Western Washington State College, 1972.

Paivio, A., \& Csapo, K. Concrete-image and verbal memory codes. Journal of Experimental Psychology, 1969, 80, 279-285.

Paivio, A., \& Csapo, K. Picture superiority in free recall: Imagery or dual coding? Cognitive Psychology, 1973, 5, 176-206.

Philipchalk, R. P., \& Rowe, E. J. Sequential and nonsequential memory for verbal and nonverbal auditory stimuli. Journal of Experimental Psychology, 1971, 91, 341-343.

RowE, E. J. Ordered recall of sounds and words in short-term memory. Bulletin of the Psychonomic Society, 1974, 4, 559-561.

Rowe, E. J., Philipchalk, R. P., \& Cake, L. J. Short-term memory for sounds and words. Joumal of Experimental Psychology, 1974, 102, 1140-1142.

Rowe, E. J., \& Rowe, W. G. Stimulus suffix effects with speech and nonspeech sounds. Memory \& Cognition, 1975. in press.

WARReN, R. M., Obusek, C. J., Farmer, R. M., \& WARren, R. P. Auditory sequence: Confusion of patterns other than speech or music. Science, 1969, 164, 586-587.

(Received for publication December 12, 1974; revision received February 3, 1975.) 